\title{
SENSEI: Direct-Detection Results on sub-GeV Dark Matter from a New Skipper CCD
}

Liron Barak, ${ }^{1}$ Itay M. Bloch, ${ }^{1}$ Mariano Cababie, ${ }^{2,3}$ Gustavo Cancelo, ${ }^{3}$ Luke Chaplinsky, ${ }^{4,5}$ Fernando Chierchie, ${ }^{3}$ Michael Crisler, ${ }^{6}$ Alex Drlica-Wagner, ${ }^{6,7,8}$ Rouven Essig, ${ }^{4}$ Juan Estrada, ${ }^{3}$ Erez Etzion, ${ }^{1}$ Guillermo Fernandez Moroni, ${ }^{3}$ Daniel Gift, ${ }^{4,5}$ Sravan Munagavalasa, ${ }^{4,5}$ Aviv Orly, ${ }^{1}$ Dario Rodrigues, ${ }^{2,3}$ Aman Singal, ${ }^{5}$ Miguel Sofo Haro, 3,9 Leandro Stefanazzi, ${ }^{3}$ Javier Tiffenberg, ${ }^{3}$ Sho Uemura, ${ }^{1}$ Tomer Volansky, ${ }^{1}$ and Tien-Tien Yu ${ }^{10}$

(SENSEI Collaboration)

\author{
${ }^{1}$ School of Physics and Astronomy, Tel-Aviv University, Tel-Aviv 69978, Israel \\ ${ }^{2}$ Department of Physics, FCEN, University of Buenos Aires and IFIBA, CONICET, Buenos Aires, Argentina \\ ${ }^{3}$ Fermi National Accelerator Laboratory, PO Box 500, Batavia Illinois 60510, USA \\ ${ }^{4}$ C.N. Yang Institute for Theoretical Physics, Stony Brook University, Stony Brook, New York 11794, USA \\ ${ }^{5}$ Department of Physics and Astronomy, Stony Brook University, Stony Brook, New York 11794, USA \\ ${ }^{6}$ Fermi National Accelerator Laboratory, PO Box 500, Batavia Illinois 60510, USA \\ ${ }^{7}$ Kavli Institute for Cosmological Physics, University of Chicago, Chicago, Illinois 60637, USA \\ ${ }^{8}$ Department of Astronomy and Astrophysics, University of Chicago, Chicago Illinois 60637, USA \\ ${ }^{9}$ Centro Atómico Bariloche, CNEA/CONICET/IB, Bariloche, Argentina \\ ${ }^{10}$ Department of Physics and Institute for Fundamental Science, University of Oregon, Eugene, Oregon 97403, USA
}

(Received 4 May 2020; revised 17 August 2020; accepted 11 September 2020;

published 20 October 2020; corrected 2 November 2020 and 20 November 2020)

\begin{abstract}
We present the first direct-detection search for sub-GeV dark matter using a new $~ 2$-gram high-resistivity Skipper CCD from a dedicated fabrication batch that was optimized for dark matter searches. Using 24 days of data acquired in the MINOS cavern at the Fermi National Accelerator Laboratory, we measure the lowest rates in silicon detectors of events containing one, two, three, or four electrons, and achieve world-leading sensitivity for a large range of sub-GeV dark matter masses. Data taken with different thicknesses of the detector shield suggest a correlation between the rate of high-energy tracks and the rate of single-electron events previously classified as "dark current." We detail key characteristics of the new Skipper CCDs, which augur well for the planned construction of the $\sim 100$-gram SENSEI experiment at SNOLAB.
\end{abstract}

DOI: 10.1103/PhysRevLett.125.171802

Introduction.-Dark matter (DM) candidates with masses below $\sim 1 \mathrm{GeV}$ are well motivated and have received increased attention in the past several years [1]. However, such DM remains poorly constrained with directdetection experiments, since the energy of the recoiling nucleus in searches for elastic DM-nucleus scattering is typically below detector thresholds. Instead, DM interactions with electrons can probe sub-GeV masses [2]. The goal of the Sub-Electron-Noise Skipper CCD Experimental Instrument (SENSEI) is to use ultralow-noise silicon Skipper charge-coupled-devices (Skipper CCDs) $[3,4]$ to probe DM down to masses of $\sim 500 \mathrm{keV}$ scattering off electrons [2,5-7] and DM down to masses of the silicon band gap of $\sim 1.2 \mathrm{eV}$ being absorbed by electrons [8-10]. SENSEI can also probe DM-nucleus scattering through the "Migdal" effect [11] down to $1 \mathrm{MeV}$ [12].

An electron that is excited from the silicon valence band to the conduction band in one of the pixels of the Skipper CCD typically creates one additional electron-hole pair (below,

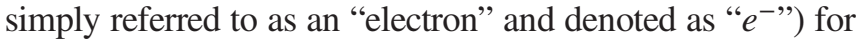

each $3.8 \mathrm{eV}$ of excitation energy above the band gap [13]. DM-electron scattering typically creates only one to a few $e^{-}$[5]. The charge in each pixel is then moved pixelto-pixel to a readout stage located in one of the corners of the Skipper CCD, where the pixel charge is measured repeatedly and nondestructively to sub-electron-noise precision [3].

The results presented in this Letter are based on data collected with high-resistivity Skipper CCDs procured in April 2019 (our previous results used prototypes [3,14,15]). The Skipper-CCD was designed at LBNL and fabricated at Teledyne DALSA Semiconductor. We describe key properties of these Skipper CCDs, and describe data that suggest a correlation between the single-electron event rate, denoted as $R_{1 e^{-}}$, and the environmental background rate as inferred from "high-energy" events, i.e., events with energies above $500 \mathrm{eV}$. We also present new DM constraints using a blinded dataset collected from February 25, 2020, to March 19, 2020, with a single Skipper CCD placed 104 m [16] underground in the MINOS cavern at Fermi National 
Accelerator Laboratory (FNAL). The Supplemental Material [17] contains additional details.

The new SENSEI Skipper CCD detectors.-The sciencegrade Skipper CCDs consist of silicon with a resistivity of $18 \mathrm{k} \Omega-\mathrm{cm}$, an active area of $9.216 \mathrm{~cm} \times 1.329 \mathrm{~cm}$, a thickness of $675 \mu \mathrm{m}$, an active mass of $1.926 \mathrm{~g}$, and 5443584 pix. No thinning process was applied to the back side to maximize the target mass and reduce fabrication cost. Each Skipper CCD has four identical amplifiers, one in each corner, which can read the entire CCD. However, the usual mode of operation is to read one quarter of the CCD consisting of 3072 rows and 443 columns of pixels. The serial register for one quadrant, which is the first row of pixels that transfers the charge to the readout stage, is along the short side of the CCD and thus consists of 443 columns. When moving charge pixel to pixel in the serial register, random $1 e^{-}$events ("spurious charge") are generated, which we measure to be $(1.664 \pm 0.122) \times 10^{-4} e^{-} /$pix and subtract from the observed $R_{1 e^{-}}$(see [17]). Each pixel has a volume of $15 \mu \mathrm{m} \times 15 \mu \mathrm{m} \times 675 \mu \mathrm{m}$ and a mass of $3.537 \times 10^{-7} \mathrm{~g}$. The DM science data are taken with the output transistor of the amplifiers turned off during exposure, although we find no evidence for amplifier-induced events that occurred in the prototype detectors [15], likely due to the improved quality of the silicon.

A silicon-aluminum pitch adapter and copper-Kapton flex cable were glued and wirebonded to the CCD. The overall width of this assembly is no larger than the width of the CCD, allowing dense packing for large-scale Skipper CCD experiments. This assembly was placed in a copper tray, where a copper leaf-spring maintains constant pressure for consistent thermal contact between the CCD and the tray (Fig. 1). The module was placed in the same vessel used for the results in [15], but with extra lead shielding placed around the vessel (see [17]), which reduced the high-energy event rate and $R_{1 e^{-}}$(see below). The CCD was operated at a temperature of $135 \mathrm{~K}$.

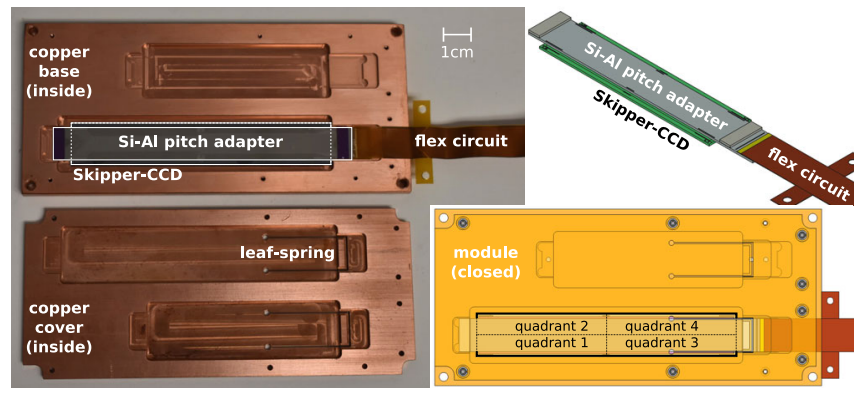

FIG. 1. A copper-Kapton flex circuit is laminated to a siliconaluminum pitch adapter that is glued and wirebonded to the Skipper CCD (top right); this is placed in a copper tray (top left), where a copper leaf-spring (bottom left) maintains constant pressure for consistent thermal contact between the CCD and the tray when closed inside the module (shown transparent, bottom right).
The readout and control systems are fully integrated in a new single-board electronics optimized for Skipper CCD sensors. This low-threshold-acquisition system [28,29] provides a flexible and scalable solution for detectors with target masses up to a few hundred grams. The root-meansquare single-sample readout noise is $2.5 e^{-}$.

Dependence of single-electron rate on environmental backgrounds. -We find evidence for a correlation between the rate of high-energy background events and $R_{1 e^{-}}$. In the DM science data (see below), which has extra lead shielding, the rate of events with $500 \mathrm{eV}$ to $10 \mathrm{keV}$ energy is 3370 DRU ( 1 DRU is 1 event $/ \mathrm{kg} /$ day $/ \mathrm{keV}$ ), while $\quad R_{1 e^{-}}=(1.594 \pm 0.160) \times 10^{-4} e^{-} / \mathrm{pix} /$ day, i.e., $(450 \pm 45) /$ g-day. This is the smallest $R_{1 e^{-}}$achieved with a semiconductor target. In one image taken without the extra lead (the "standard" shield), we find $R_{1 e^{-}}=\left(7.555_{-2.562}^{+3.286}\right) \times 10^{-4} e^{-} / \mathrm{pix} /$ day. Three additional standard-shield images (but taken with the amplifier voltages turned on during exposure) show $R_{1 e^{-}}=$ $\left(4.302_{-1.426}^{+1.743}\right) \times 10^{-4} e^{-} / \mathrm{pix} /$ day, so that, averaged over the four images, $R_{1 e^{-}}=\left(5.312_{-1.277}^{+1.490}\right) \times 10^{-4} e^{-} /$pix/day, i.e., $1492_{-361}^{+421} /$ g-day. The combined standard-shield highenergy background rate is 9700 DRU; see Fig. 2 and [17] for more details. The origin of the $1 e^{-}$events requires further study. We have insufficient data to measure $R_{2 e^{-}}$for the standard-shield case. The high-energy event spectra are shown in the Supplemental Material [17].

Data collection.-We collect blinded data for constraining DM that produces events with $\leq 4$ electrons. We expose the Skipper CCD for 20 hours, and then read each quadrant through one amplifier with 300 samples per pixel. We refer to one such exposure and readout as an "image." We took 22 images of DM science data before a mandatory shutdown. All charge on the CCD is erased

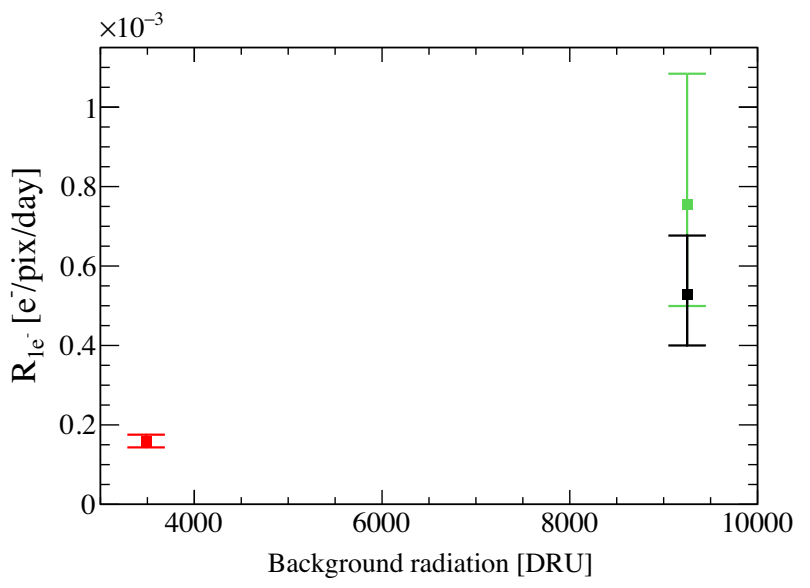

FIG. 2. Single-electron event rate, $R_{1 e^{-}}$(after subtracting the spurious charge) versus the rate of events with energies $500 \mathrm{eV}$ to $10 \mathrm{keV}$ with extra shielding (red) and without extra shielding for one image with the amplifier off during exposure (green) and when combined with three images with the amplifier on during exposure (black). 
TABLE I. Efficiencies and number of events containing $1 e^{-}, 2 e^{-}, 3 e^{-}$, or $4 e^{-}$events that pass the masking cuts for the $1 e^{-}, 2 e^{-}, 3 e^{-}$, and $4 e^{-}$analysis, respectively. The charge diffusion cut assumes the DM generates single-pixel events for $N_{e}=2 e^{-}$or contiguous multi-pixel events for $N_{e}=3 e^{-}$or $N_{e}=4 e^{-}$. The total efficiency is the fraction of pixels that pass all cuts, while the effective ("Eff.") efficiency is exposure corrected (since each pixel has a unique exposure) and, for $3 e^{-}$and $4 e^{-}$, includes a geometric efficiency. The bottom three rows, respectively, list the efficiency-corrected exposure, the number of observed events after cuts, and the $90 \%$ C.L. limits. ${ }^{(*)}$ For $1 e^{-}$, we list the number after subtracting $0 e^{-}$(adding $\left.1 e^{-}\right)$events that are misclassified as $1 e^{-}\left(0 e^{-}\right.$or $\left.2 e^{-}\right)$; the quoted limit is after subtracting the spurious charge.

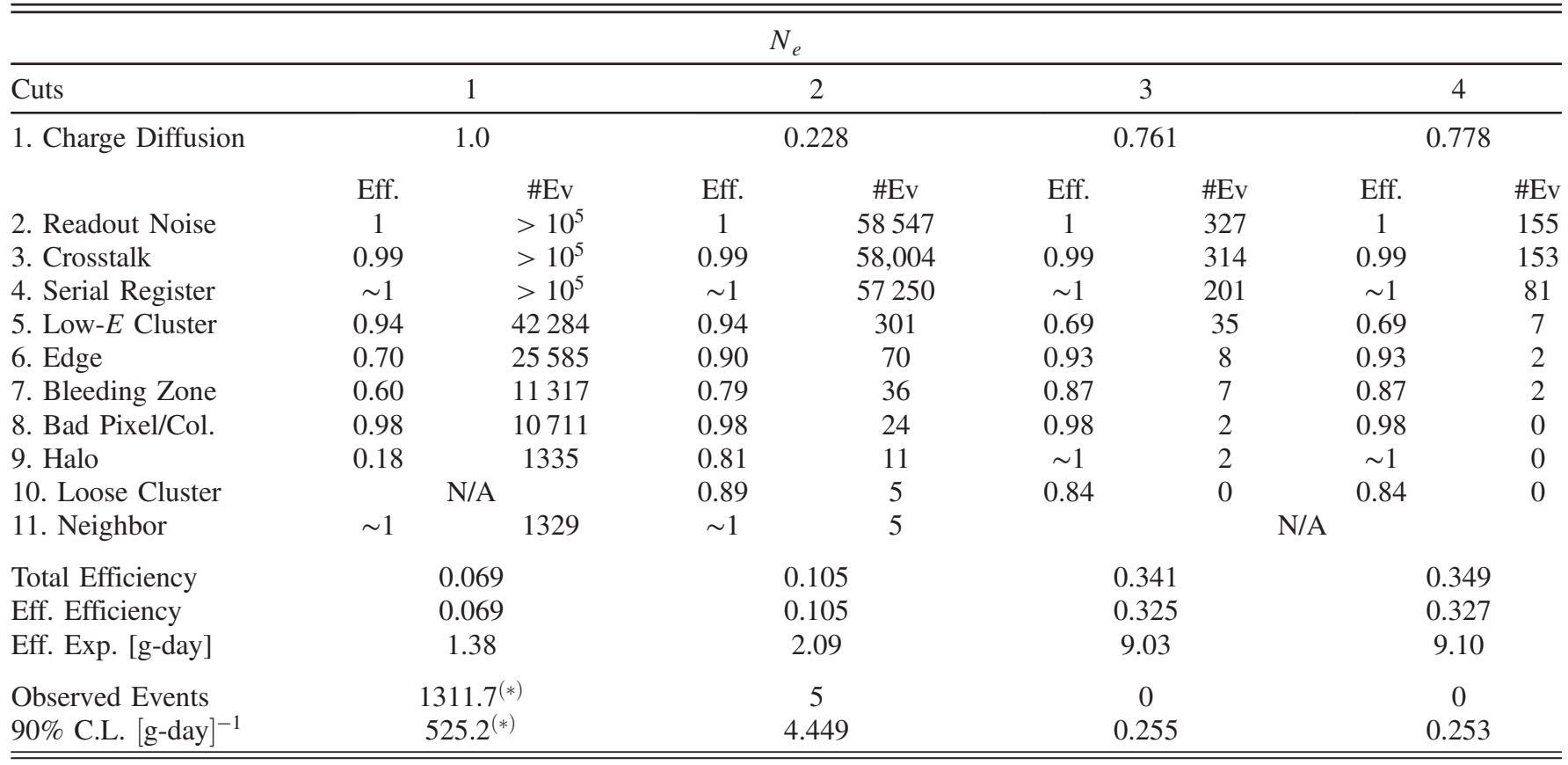

before taking a new image. The read time per sample is $42.825 \mu \mathrm{s}$, while the readout time of the entire active area is 5.153 hours. Commissioning data, consisting of (7) 20-hour-exposure images, were used to determine the data quality cuts. During commissioning, "quadrant 1 " and "quadrant 2" showed excellent performance, with a rootmean square noise of $0.146 e^{-}$and $0.139 e^{-}$(with negligible error bars), respectively. "Quadrant 4" had an excessively high charge transfer inefficiency (consistent with a disconnected serial register clock) and its data were discarded. In addition, "quadrant 3" (next to quadrant 4 on the short CCD side), with a noise of $0.142 e^{-}$, had an excess of $1 e^{-}$ events in the entire quadrant, but especially in the first $\sim 100$ columns, consistent with possible blackbody radiation from the surrounding warm vessel leaking onto that part of the cold CCD through the leaf-spring slots (Fig. 1). Before unblinding, we thus discarded quadrant 3 's data for the $1 e^{-}$ and $2 e^{-}$analyses; however, we include its columns 93 to 443 for the $3 e^{-}$and $4 e^{-}$analyses to increase our exposure and since the expected probability of a single spurious $3 e^{-}$ event is at the percent level. The total exposure (before cuts) of the DM search data is $19.93 \mathrm{~g}$-day for the $1 e^{-}$and $2 e^{-}$ analyses, and $27.82 \mathrm{~g}$-day for the $3 e^{-}$and $4 e^{-}$analyses.

Data analysis.-We perform four analyses: on $1 e^{-}$ events, on single-pixel $2 e^{-}$events, and on events consisting of a contiguous set of pixels containing a total of $3 e^{-}$or $4 e^{-}$. Most event selection criteria are common to the four analyses, but there are important differences, mostly because the $1 e^{-}$and (to a lesser extent) the $2 e^{-}$analyses are not exposure limited. Due to nonzero noise, we define a $\left(1 e^{-}\right.$, $\left.2 e^{-}, 3 e^{-}, 4 e^{-}\right)$pixel to have a measured charge in the range $[(0.63,1.63],(1.63,2.5],(2.5,3.5],(3.5,4.5]] e^{-}$, respectively. Pixels with $\geq 1 e^{-}$have a measured charge of $>0.63 e^{-}$. A "cluster" is defined as a contiguous set of neighboring pixels that each have a measured charge of $>0.63 e^{-}$. Given a pixel, a "neighboring" pixel is one of the eight adjacent pixels. The charge of a cluster is the sum of the pixel charges. For counting the final number of $1 e^{-}$events, we use a Gaussian fit to remove $0 e^{-}$(add $1 e^{-}$) events that have a measured charge $>0.63 e^{-}\left(\leq 0.63 e^{-}\right.$or $\left.>2.5 e^{-}\right)$.

We summarize next the event-selection criteria (see Table I). Additional details are in [17].

Charge diffusion: We account for the efficiency for DM events with $\geq 2 e^{-}$to be spread out over more than one pixel due to charge diffusion. The efficiency for detecting $1 e^{-}$ events is unity [30].

Readout noise: We veto images in which the readout noise is $30 \%$ larger than the expected readout noise. No such images are observed.

Crosstalk: A pixel is masked if it is read at the same time as another pixel containing $>700$ electrons.

Serial register hit: We remove isolated horizontal lines of charge, which indicate a background event that hit the serial register during readout. 
Low-energy cluster: We observe disjoint groupings of $\geq 2 e^{-}$events that are too close to be a coincidence. For the $1 e^{-}$and $2 e^{-}\left(3 e^{-}\right.$and $\left.4 e^{-}\right)$analyses, we thus remove 4 (20) pixels in all directions from all pixels that are part of a cluster containing at least $5 e^{-}\left(2 e^{-}\right)$. We do not remove the pixels of the cluster itself.

Edge mask: We remove 60 (20) pixels around all edges of a quadrant for the $1 e^{-}\left(\geq 2 e^{-}\right)$analyses, which corresponds to the halo mask (described below) for any possible high-energy events occurring just outside of the quadrant.

Bleeding zone mask: To avoid spurious events from charge-transfer inefficiencies, we mask 100 (50) pixels upstream in the vertical and horizontal direction of any pixel containing more than $100 e^{-}$for the $1 e^{-}\left(\geq 2 e^{-}\right)$ analyses. This distance is doubled for columns where we observe a high bleeding rate.

Bad pixels and bad columns: We further limit the impact of defects that cause charge leakage or charge-transfer inefficiencies by identifying and masking pixels and columns that have a significant excess of charge.

Halo mask: Pixels with more than $100 e^{-}$, from highenergy background events, correlate with an increased rate of low-energy events in nearby pixels. We observe a monotonic decrease in $R_{1 e^{-}}$as a function of the radial distance, $R$, from pixels with a large charge. We mask pixels out to $R=60$ pix $(R=20$ pix $)$ for the $1 e^{-}\left(\geq 2 e^{-}\right)$analyses.

Loose cluster mask: We find a correlation between the number of $1 e^{-}$and $2 e^{-}$events in regions of size $\sim 10^{3} \mathrm{pix}^{2}$. Since there is no reason for a $2 e^{-}$DM event to be spatially correlated with an excess of $1 e^{-}$events, we mask regions with an excess of $1 e^{-}$events. We apply this mask only for the $\geq 2 e^{-}$analyses.

Neighbor mask: For the $1 e^{-}$and $2 e^{-} \mathrm{DM}$ analyses only, we require the DM signal to be contained in a single pixel and only select pixels whose eight neighboring pixels are empty. We thus mask all pixels that have a neighboring pixel with $\geq 1 e^{-}$.

The efficiencies of, and number of events passing, these selection cuts are given in Table I, which also shows the number of observed events and the inferred $90 \%$ confidence level (C.L.) upper limits on the rates. We assume that a DM signal is uniformly distributed across the CCD so that a cut's efficiency on a DM signal is proportional to the loss in exposure from that cut.

Dark matter results.-The results for the four analyses are as follows: (i) $1 e^{-}$: from the observed $R_{1 e^{-}}$of $(3.363 \pm$ $0.094) \times 10^{-4} e^{-} / \mathrm{pix} /$ day, we subtract the (exposure independent) spurious charge contribution of (1.664 \pm $0.122) \times 10^{-4} e^{-} /$pix, to arrive at a $R_{1 e^{-}}$of $(1.594 \pm$ $0.160) \times 10^{-4} e^{-} / \mathrm{pix} /$ day, or $(450 \pm 45)$ events/g-day, where the errors have been added in quadrature. For calculating a DM limit below, we conservatively take the 1311.7 observed $1 e^{-}$events and subtract the $2 \sigma$ lower limit on the number of expected spurious-charge events $(649-2 \times 47.5=554$ events $)$, arriving at $\sim 7581 e^{-}$events. The known contributions to $R_{1 e^{-}}$that we do not subtract are environmental backgrounds and dark current (from thermal excitations). Of these, we expect the dark current contribution to be more than an order of magnitude lower than the observed $R_{1 e^{-}}$[17]. (ii) $2 e^{-}$: the five observed single-pixel $2 e^{-}$events imply $R_{2 e^{-}}=2.399$ events/g-day, with a $90 \%$ C.L. upper limit of $R_{2 e^{-}}=$ $4.449 /$ g-day $(\simeq 0.051 \mathrm{~Hz} / \mathrm{kg})$. This is more than two orders of magnitude lower than previous measurements of $R_{2 e^{-}}$in solid-state detectors, and strongly disfavors a possible DM interpretation for the excess events observed in previous experiments [31]. (iii) $3 e^{-}$and $4 e^{-}$: we observe zero $3 e^{-}$and $4 e^{-}$clusters to find $90 \%$ C.L. upper limits on $R_{3 e^{-}}$of $0.255 / \mathrm{g}$-day and on $R_{4 e^{-}}$of $0.253 / \mathrm{g}$-day.

Figure 3 shows the observed spectra of events after all cuts. We use these data to constrain DM that scatters off electrons [2,5], DM that is absorbed by electrons [8-10,32-34]), and DM that scatters off nuclei through the Migdal effect [12]. Current estimates of the Migdal effect at low recoil energies and especially for DM masses $\lesssim 10 \mathrm{MeV}$ are uncertain [12], so the resulting limits on DM-nucleus scattering should be viewed as approximate only. We assume an electron with recoil energy $E_{e}$ generates $\left[1+\right.$ Floor $\left.\left(E_{e}-1.2 \mathrm{eV}\right) / \varepsilon\right] e^{-}$; new measurements at $\sim 6 \mathrm{keV}$ find $\varepsilon=3.75 \mathrm{eV}$ [35], but we will take $\varepsilon=$ $3.8 \mathrm{eV}$ for consistency with other DM results in the literature and since the precise extrapolation to low energies remains uncertain. We conservatively ignore Fano-factor fluctuations for scattering, while for absorption we follow [9]. We assume a local DM density of $\rho_{\mathrm{DM}}=0.3 \mathrm{GeV} / \mathrm{cm}^{3}$ [36], a standard isothermal Maxwellian velocity distribution [37] with a DM escape velocity of $600 \mathrm{~km} / \mathrm{s}$, a mean local DM velocity of $230 \mathrm{~km} / \mathrm{s}$, and an average Earth velocity of $240 \mathrm{~km} / \mathrm{s}$. Figure 4 shows the resulting "SENSEI@MINOS" 90\% C.L. that combines the four analyses. We use a likelihood-ratio test based on [38], with a toy Monte Carlo (instead of the asymptotic approximation) to compute the distribution of the $q_{\mu}$ statistics used for the calculation of the $p$ value.

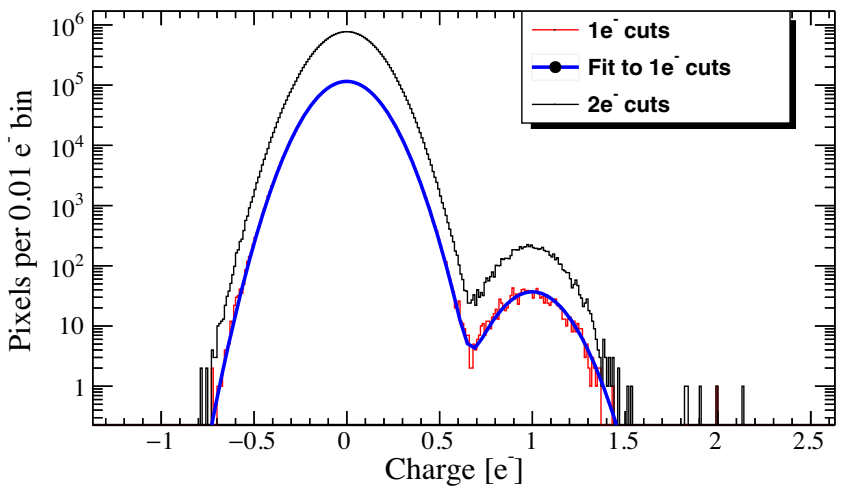

FIG. 3. The pixel charge spectra (after selection cuts) used for the $1 e^{-}$and $2 e^{-}$analyses. A double-Gaussian fit is shown for the spectrum with $1 e^{-}$cuts. There are no $3 e^{-}$or $4 e^{-}$events. 

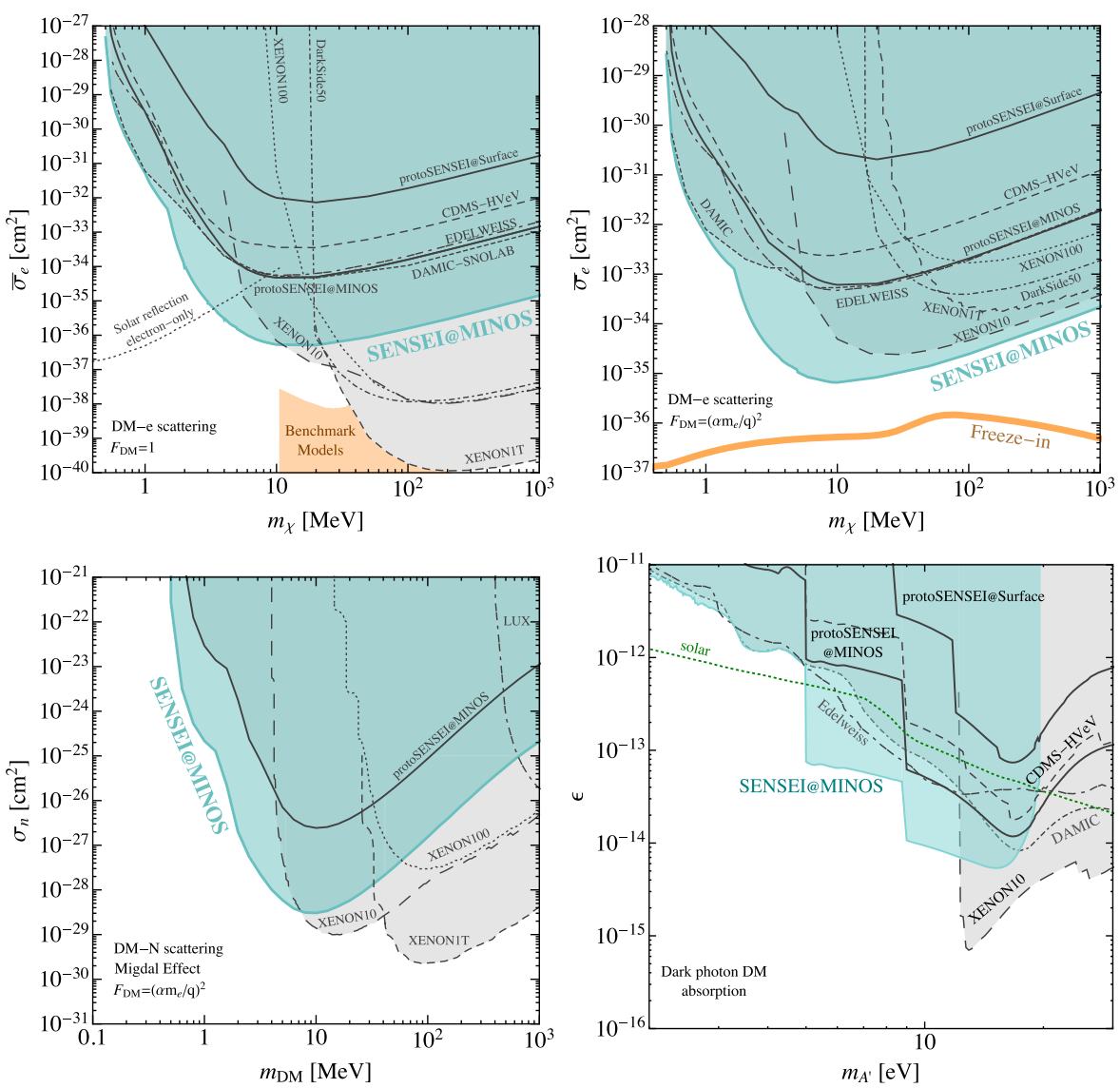

FIG. 4. $90 \%$ C.L. constraints (cyan solid line) on: DM $-e^{-}$cross section, $\bar{\sigma}_{e}$, versus DM mass, $m_{\chi}$, for two DM form factors, $F_{\mathrm{DM}}(q)=1$ (top left) and $F_{\mathrm{DM}}(q)=\left(\alpha m_{e} / q\right)^{2}$ (top right); DM-nucleus cross section, $\bar{\sigma}_{n}$, for a light mediator (bottom left); and the kinetic-mixing parameter, $\epsilon$, versus the dark-photon mass, $m_{A^{\prime}}$, for dark-photon-DM absorption (bottom right). Constraints are shown on DM- $e^{-}$scattering also from the SENSEI prototype [14,15], XENON10/100 [39], DarkSide-50 [40], EDELWEISS [41], CDMS-HVeV [42], XENON1T [43], DAMIC [44], solar reflection (assuming DM couples only to $e^{-}$) [45]; constraints on DM-nucleus scattering from SENSEI, XENON10/100/1T [12] and LUX [46]; and constraints on absorption from SENSEI [14,15], DAMIC [44,47], EDELWEISS [41], XENON10/100, CDMSlite [9], and the Sun [9,48,49]. Orange regions are combined benchmark model regions for heavy $[2,5$, $50-54]$ and light $[2,5,55,56]$ mediators.

For DM-electron scattering via a heavy (light) mediator, SENSEI@MINOS provides world-leading constraints for $m_{\chi} \sim 500 \mathrm{keV}-10 \mathrm{MeV} \quad\left(m_{\chi} \gtrsim 500 \mathrm{keV}\right)$. For DMnucleus scattering through a light mediator and for DM absorption on electrons, SENSEI@MINOS provides world-leading constraints for $m_{\chi} \sim 600 \mathrm{keV}-5 \mathrm{MeV}$ and $m_{\chi} \sim 1.2 \mathrm{eV}-12.8 \mathrm{eV}$, respectively.

Outlook.-The SENSEI Collaboration is in the process of packaging and testing $~ 75$ sensors from the same batch as the one used for this work. Up to $\sim 50(\sim 100 \mathrm{~g})$ of packaged science-grade Skipper CCDs will be deployed in a phased approach inside a low radiation shield currently being built at SNOLAB. We expect that the low radiation environment will translate to even lower $R_{1 e^{-}}$. We plan to commission the first batch of sensors (tens of grams) over the next year. We plan to accumulate $\sim 100$ g-years of exposure over one to two years.
We thank Kyle Cranmer for useful discussions on the limit calculation. We are grateful for the support of the Heising-Simons Foundation under Grant No. 79921. R. E. also acknowledges support from DOE Grant DESC0017938 and Simons Investigator in Physics Award No. 623940. This work was supported by Fermilab under DOE Contract No. DE-AC02-07CH11359. The work of T. V. and E. E. is supported by the I-CORE Program of the Planning Budgeting Committee and the Israel Science Foundation (Grant No. 1937/12). T. V. is further supported by the European Research Council (ERC) under the EU Horizon 2020 Programme (ERC- CoG-2015 -Proposal No. 682676 LDMThExp), and a grant from The Ambrose Monell Foundation, given by the Institute for Advanced Study. The work of S. U. is supported in part by the Zuckerman STEM Leadership Program. I. B. is grateful for the support of the Alexander Zaks Scholarship, The Buchmann Scholarship, and the Azrieli Foundation. This 
manuscript has been authored by Fermi Research Alliance, LLC under Contract No. DE-AC02-07CH11359 with the U.S. Department of Energy, Office of Science, Office of High Energy Physics. The CCD development work was supported in part by the Director, Office of Science, of the U.S. Department of Energy under Contract No. DE-AC0205CH11231. The United States Government retains and the publisher, by accepting the Letter for publication, acknowledges that the United States Government retains a nonexclusive, paid-up, irrevocable, worldwide license to publish or reproduce the published form of this manuscript, or allow others to do so, for United States Government purposes.

[1] M. Battaglieri et al., in U.S. Cosmic Visions: New Ideas in Dark Matter (2017).

[2] R. Essig, J. Mardon, and T. Volansky, Phys. Rev. D 85, 076007 (2012).

[3] J. Tiffenberg, M. Sofo-Haro, A. Drlica-Wagner, R. Essig, Y. Guardincerri, S. Holland, T. Volansky, and T.-T. Yu, Phys. Rev. Lett. 119, 131802 (2017).

[4] G. F. Moroni, J. Estrada, G. Cancelo, S. Holland, E. Paolini, and H. Diehl, Exp. Astron. 34, 43 (2012).

[5] R. Essig, M. Fernandez-Serra, J. Mardon, A. Soto, T. Volansky, and T.-T. Yu, J. High Energy Phys. 05 (2016) 046.

[6] S. K. Lee, M. Lisanti, S. Mishra-Sharma, and B. R. Safdi, Phys. Rev. D 92, 083517 (2015).

[7] P. W. Graham, D. E. Kaplan, S. Rajendran, and M. T. Walters, Phys. Dark Universe 1, 32 (2012).

[8] H. An, M. Pospelov, J. Pradler, and A. Ritz, Phys. Lett. B 747, 331 (2015).

[9] I. M. Bloch, R. Essig, K. Tobioka, T. Volansky, and T.-T. Yu, J. High Energy Phys. 06 (2017) 087.

[10] Y. Hochberg, T. Lin, and K. M. Zurek, Phys. Rev. D 95, 023013 (2017).

[11] M. Ibe, W. Nakano, Y. Shoji, and K. Suzuki, J. High Energy Phys. 03 (2018) 194.

[12] R. Essig, J. Pradler, M. Sholapurkar, and T.-T. Yu, Phys. Rev. Lett. 124, 021801 (2020).

[13] V. S. Vavilov, Sov. Phys. Usp. 4, 761 (1962).

[14] M. Crisler, R. Essig, J. Estrada, G. Fernandez, J. Tiffenberg, M. S. haro, T. Volansky, and T.-T. Yu (SENSEI Collaboration), Phys. Rev. Lett. 121, 061803 (2018).

[15] O. Abramoff, L. Barak, I. M. Bloch, L. Chaplinsky, M. Crisler et al. (SENSEI Collaboration), Phys. Rev. Lett. 122, 161801 (2019).

[16] P. Adamson et al. (MINOS Collaboration), Phys. Rev. D 77, 072002 (2008).

[17] See the Supplemental Material at http://link.aps.org/ supplemental/10.1103/PhysRevLett.125.171802 for additional details of our analysis and additional Refs. [18-27].

[18] J. R. Janesick, Scientific Charge-Coupled Devices (SPIE Press, 2001), Vol. 83, https://doi.org/10.1117/3.374903.

[19] M. S. Haro, G. Cancelo, G. F. Moroni, X. Bertou, J. Tiffenberg, E. Paolini, and J. Estrada, in 2016 Argentine Conference of Micro-Nanoelectronics, Technology and Applications (CAMTA) (IEEE, New York, 2016), pp. 1116, https://ieeexplore.iee.org/document/7574083.

[20] SENSEI Collaboration (to be published).
[21] S. E. Holland, D. E. Groom, N. P. Palaio, R. J. Stover, and M. Wei, IEEE Trans. Electron Devices 50, 225 (2003).

[22] A. Aguilar-Arevalo, D. Amidei, X. Bertou, M. Butner, G. Cancelo et al. (DAMIC Collaboration), Phys. Rev. D 94, 082006 (2016).

[23] A. Aguilar-Arevalo, X. Bertou, C. Bonifazi, G. Cancelo, A. Castaneda et al. (CONNIE Collaboration), Phys. Rev. D 100, 092005 (2019).

[24] M. S. Haro, G. F. Moroni, and J. Tiffenberg, IEEE Trans. Electron Devices 1 67, 1993 (2020).

[25] G. M. Bernstein et al., Publ. Astron. Soc. Pac. 129, 114502 (2017).

[26] B. E. Burke and S. A. Gajar, IEEE Trans. Electron Devices 38, 285 (1991).

[27] K. Krishnamoorthy and J. Thomson, J. Stat. Plann. Inference 119, 23 (2004).

[28] G. F. Moroni et al., in 2019 Argentine Conference on Electronics (CAE) (IEEE, New York, 2019). pp. 86-91, https://ieeexplore.iee.org/abstract/document/8709274.

[29] G. Cancelo et al., arXiv:2004.07599.

[30] M. H. Fabricius, C. J. Bebek, D. E. Groom, A. Karcher, and N. A. Roe, in Proceedings of the SPIE, Society of PhotoOptical Instrumentation Engineers (SPIE) Conference Series Vol. 6068, edited by M. M. Blouke (2006), pp. 144-154.

[31] N. Kurinsky, D. Baxter, Y. Kahn, and G. Krnjaic, Phys. Rev. D 102, 015017 (2020).

[32] E. Saloman and J. Hubbel (1986), https://inis.iaea.org/ search/search.aspx?orig_q=RN:18048512.

[33] X-ray interactions with matter, http://henke.lbl.gov/optical_ constants/.

[34] D. F. Edwards, in Handbook of Optical Constants of Solids, edited by E. D. Palik (Academic Press, Boston, 1985), pp. 547-569.

[35] D. Rodrigues et al., arXiv:2004.11499.

[36] J. Bovy and S. Tremaine, Astrophys. J. 756, 89 (2012).

[37] J. D. Lewin and P. F. Smith, Astropart. Phys. 6, 87 (1996).

[38] G. Cowan, K. Cranmer, E. Gross, and O. Vitells, Eur. Phys. J. C 71, 1554 (2011); 73, 2501(E) (2013).

[39] R. Essig, T. Volansky, and T.-T. Yu, Phys. Rev. D 96, 043017 (2017).

[40] P. Agnes et al. (DarkSide Collaboration), Phys. Rev. Lett. 121, 111303 (2018).

[41] Q. Arnaud et al. (EDELWEISS Collaboration), arXiv:2003.01046.

[42] R. Agnese et al. (SuperCDMS Collaboration), Phys. Rev. Lett. 121, 051301 (2018).

[43] E. Aprile et al. (XENON Collaboration), Phys. Rev. Lett. 123, 251801 (2019).

[44] A. Aguilar-Arevalo, D. Amidei, D. Baxter, G. Cancelo, B. A. CervantesVergara et al. (DAMIC Collaboration), Phys. Rev. Lett. 123, 181802 (2019).

[45] H. An, M. Pospelov, J. Pradler, and A. Ritz, Phys. Rev. Lett. 120, 141801 (2018); 121, 259903(E) (2018).

[46] D. Akerib et al. (LUX Collaboration), Phys. Rev. Lett. 122, 131301 (2019).

[47] A. Aguilar-Arevalo, D. Amidei, X. Bertou, M. Butner, G. Cancelo et al. (DAMIC Collaboration), Phys. Rev. Lett. 118, 141803 (2017).

[48] H. An, M. Pospelov, and J. Pradler, Phys. Lett. B 725, 190 (2013). 
[49] J. Redondo and G. Raffelt, J. Cosmol. Astropart. Phys. 08 (2013) 034.

[50] C. Boehm and P. Fayet, Nucl. Phys. B683, 219 (2004).

[51] T. Lin, H.-B. Yu, and K. M. Zurek, Phys. Rev. D 85, 063503 (2012).

[52] Y. Hochberg, E. Kuflik, T. Volansky, and J. G. Wacker, Phys. Rev. Lett. 113, 171301 (2014).

[53] E. Kuflik, M. Perelstein, N. R.-L. Lorier, and Y.-D. Tsai, J. High Energy Phys. 08 (2017) 078.

[54] R. T. D’Agnolo, D. Pappadopulo, J. T. Ruderman, and P.-J. Wang, Phys. Rev. Lett. 124, 151801 (2020).
[55] X. Chu, T. Hambye, and M. H. Tytgat, J. Cosmol. Astropart. Phys. 05 (2012) 034.

[56] C. Dvorkin, T. Lin, and K. Schutz, Phys. Rev. D 99, 115009 (2019).

Correction: The omission of a support statement in the Acknowledgments section has been fixed.

Second Correction: A sentence about the Skipper CCD was added after the first sentence in the third paragraph in the Introduction. 